\title{
Quand une femme écrit en homme
}

When a woman writes in man

\section{Hélène Lenz}

\section{(2) OpenEdition}

\section{Journals}

Édition électronique

URL : https://journals.openedition.org/ceb/3988

DOI : $10.4000 /$ ceb.3988

ISSN : 2261-4184

Éditeur

INALCO

\section{Édition imprimée}

Pagination : 251-265

ISBN : 978-2-85837-205-4

ISSN : 0290-7402

\section{Référence électronique}

Hélène Lenz, "Quand une femme écrit en homme », Cahiers balkaniques [En ligne], 41 | 2013, mis en ligne le 19 mai 2013, consulté le 06 juillet 2021. URL : http://journals.openedition.org/ceb/3988 ; DOI : https://doi.org/10.4000/ceb.3988

Ce document a été généré automatiquement le 6 juillet 2021.

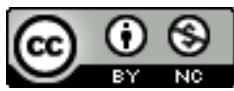

Cahiers balkaniques est mis à disposition selon les termes de la Licence Creative Commons Attribution - Pas d'Utilisation Commerciale 4.0 International. 


\title{
Quand une femme écrit en homme
}

\author{
When a woman writes in man
}

Hélène Lenz

1 Maria Banus (Bucarest 1914- Paris 1999) dont le Journal de fin de guerre, paru en 1977 à Bucarest, est présenté ici, n'est pas une anonyme de la «lutte antifasciste ». Assez connue en dehors de son pays d'origine bien qu'oubliée ou écartée par l'historiographie postcommuniste, elle voit aujourd'hui son œuvre, sinon son expérience, réestimée ${ }^{1}$. Poète, essayiste, traductrice membre dès 1945 de la SSR (Societatea Scriitorilor Români) Société des Écrivains roumains) ${ }^{2}$, elle a fait partie aussi de l'Uniunea Scriitorilor din R.P.R/ Union des Écrivains de la République populaire de Roumanie. Sa réussite roumaine est avérée jusqu'à son exil en France (1987) où elle sera traduite, l'année suivant son arrivée, par Alain Bosquet aux côtés de Anna Akhmatova, Gabriela Mistral (prix Nobel de littérature 1945), Else Laske Schüller avant de recevoir en 1989 le prix Gottfried von Herder. Auparavant, sa carrière d'auteur avait franchi plusieurs degrés d'un cursus honorum communiste: Prix George Cosbuc de l'Académie de la République populaire roumaine (1949), Prix d'État (1951), Médaille d'Or du Festival mondial de la Jeunesse et des Étudiants de Varsovie (1955), Prix spécial de l'Union des Écrivains (1986) et elle avait été décorée en 1964 de l'Ordre du Travail de $1^{\text {re }}$ classe ${ }^{3}$. Entre 1949 et 1990, Maria Banus aura fait paraitre en Roumanie 23 recueils poétiques, partiellement traduits en France par Guillevic. Elle aura encore publié en 1978 à Bucarest «Sub Camuflaj/ Sous camouflage ", extraits de son Journal : 1943-44.

2 Ce livre intéresse aujourd'hui à la fois pour sa valeur de document sur une conscience féminine consciente de témoigner sur la guerre, dans et à travers son sexe, et pour ce qu'il révèle des paris proposés à une militante politique d'origine bourgeoise dans le nord des Balkans avant-guerre, pendant la guerre et après guerre - une fois les frontières verrouillées par le rideau de fer. La narratrice y témoigne de son attachement à sa langue d'expression, le roumain, à son identité roumaine. Elle insiste parallèlement sur une identité juive dont elle découvre alors les implications culturelles existentielles mettant en jeu son identité d'artiste au point de la modifier pour le reste de sa vie. Implicitement et paradoxalement, cette démarche tantôt naïve, tantôt studieuse (références à la Kabbale), tantôt focalisée sur la notation 
d'informations sur les femmes (notamment déportées) fait échapper le Journal au cadre géographique de la Roumanie, des Balkans, jusqu'à lui faire laisser rejoindre des courants occidentaux, l'après 68 ou la beat generation américaine. Le titre donné au Journal, "Sous camouflage", peut renvoyer aux possibilités d'accomplissement clandestines lors d'une crise politique privant un quidam d'existence légale, l'acculant à une seule possibilité de survie (ici l'adhésion à un réseau communiste). Sans doute sa publication a-t-elle été autorisée lors du durcissement de la dictature Ceaucescu pour son exemplarité. Dans les années 1975-80, la dégradation des conditions de vie roumaines a mené des quantités croissantes de Roumains vers l'émigration légale ou illégale. L'exemple de créativité, de tonicité donné par le Journal a pu être crédité d'une valeur dissuasive ou incitative. N'excluons pas que l'auteur ait joué de cette manipulation par l'édition/ censure en «forçant un créneau » de l'historiographie officielle nationale, esquissé dans sa poésie des années 50 , celui des déportations en Transnistrie, sur lesquelles la lumière n'a été vraiment faite qu'après la chute du mur de Berlin. Elle a pu vouloir aussi consciemment contribuer à une chronique du quotidien sur la Résistance roumaine, sujet annexé par l'idéologie durant sept années d'occupation soviétique, 45 ans de communisme et "généralement ignoré de l'historiographie occidentale grand public " aujourd'hui encore. Si l'offensive de l'Armée rouge contre les Roumains et les Allemands (1944) est connue, la modification des alliances dès l'été 1943 (le Journal commence le 19 mars 1943), sous la présidence secrète du roi Michel, a réuni des groupes politiques comprenant communistes et leaders d'anciens partis démocratiques. Cette réalité implique des présupposés de mentalité 4 . Publié à 20000 exemplaires ( $1^{\text {re }}$ édition) le livre présente aussi une version informelle acceptée sous la dictature des conditions dans lesquelles une telle résistance a pu germer ${ }^{5}$.

\section{Femme et Femmes}

Révélée à 14 ans, en 1928, par le pamphlétaire Tudor Arghezi, éditeur de revues, Maria Banus a publié en 1937 Țara fetelor/ le Pays des jeunes filles, un recueil de poèmes influencé par l'expressionnisme allemand. Le livre témoignait d'une exaltation peu courante de la sensualité féminine. Sans doute le Bucarest d'avant-guerre ne s'érige-t-il pas en parangon du puritanisme, avec sa réputation de lupanar des Balkans, sa Cour royale germano-anglaise agitée de rumeurs (la reine Marie adultère délaissée par son époux Carol II, roi allemand préparant son départ du pays avec sa maitresse juive), ses littérateurs d'extrême droite que tourmentent la présence soviétique à l'Est et l'européanisation d'une capitale dont les femmes se libèrent en se débauchant (Mircea Eliade condamné pour pornographie en 1936 pour "Mlle Christina " qui met en scène une aristocrate nymphomane, fustigera les garçonnes franco-américanophiles ruinant la vision chrétienne bourgeoise du mariage dans "Noces au Paradis»). Tributaire de ce contexte dépassé par les drames d'une nouvelle entrée en guerre, Sub camuflaj dispense cependant anecdotes, observations, témoignant d'une perception du monde et de l'existence à partir de positions exprimées ultérieurement dans « Éternel féminin » :

Être femme - être homme - mais surtout recevoir l'univers depuis l'AUTRE angle de vues biologique. Être réceptacle! - souriez, moqueurs! - vous place dans d'autres relations, existentielles et sociales avec le monde. Même en faisant abstraction de l'Histoire, de l'infériorité imposée par la loi ou les préjugés (...) le destin de la femme, sa sensibilité, sa Weltanschaung seront toujours marquées d'un signe 
distinct. Ce signe n'est ni un moins comme l'assurent les misogynes ni un plus comme le croient dans leur réflexion narcissique les amazones féministes. Nous sommes l'AUTRE (...). Pour la naissance et la mort, nous sommes frères de l'homme. Mais nous sommes ses adversaires, ses ennemis implacables sur le terrain de lutte de la sexualité, des passions. Je fais à nouveau abstraction du plan social où l'homme détient aujourd'hui la supériorité - de droit parfois, de fait le plus souvent-. J'imagine un monde où sous l'angle de la promotion sociale toute inégalité des sexes serait abolie. Nous resterions quand même l'AUTRE. Par bonheur. Sans la pluralité masculin-féminin, le monde serait insipide ! 6

Les amies, les connaissances rendant visite à la diariste ou l'invitant en retour sont nombreuses. Elles donnent lieu à des portraits fournissant une information "sexuée " sur une actualité, dont le caractère insupportable, s'accentue en fin du volume (accélération d'arrestations/ déportations en Transnistrie, multiplication d'informations sur ces camps avant le bombardement de Bucarest sur lequel l'auteur prend des notes). Le Journal évoque, au début, des mondanités qu'il épingle avec amusement. Intellectuelle, écrivaine déjà connue, personnalité atypique (elle a étudié le droit aux côtés des lettres), Maria Banus n'est pas familiarisée avec le mode de vie des « inactives » de classe supérieure ou moyenne. «Pour la première fois de ma vie, je me rends à un thé de dames" (20 mars 1943) ${ }^{7}$. L'invitation est agréée en vue de résultats renforçant un réseau : l'engagement communiste de femmes se réduit peut-être à ce stade à la pratique d'activités caritatives (comme ultérieurement, lors de la fréquentation d'un foyer juif). «Peut-être réussirai-je à me faire des relations, à rassembler des fonds, me suis-je dit en acceptant l'invitation de Frou-Frou " ${ }^{8}$. Le temps perdu est résumé en quatre rubriques prouvant l'inanité de conversations sur le vol des bijoux de la famille 0 ., les deux derniers scandales mondains, les jeux de société, et des adultères réussis et ratés ${ }^{9}$. Les cérémonies de la frange sociale le plus souvent évoquée dans le Journal, les petits bourgeois citadins, suscitent davantage d'attention. Le " mariage civil de Ana $»^{10}$ annonce de nouveaux virages politiques et une mise à distance qui va s'aggraver. Une réunion familiale chez Tanti Lucica présente au lecteur deux grandmères, celle d'Anton (mari de Maria) ${ }^{11}$, et la sienne propre dont la réussite sera évoquée en fin du Journal (couturière à succès, elle aurait été invitée à suivre à Paris Hélène Vacaresco, écrivaine un temps fiancée avec le Prince Ferdinand). Enfin la mère de Maria Banus ${ }^{12}$, pâle Bovary illustrant chances et malchances d'une bourgeoisie juive émancipée, rappelle aussi le profil de la mère d'une Hannah Arendt en Allemagne. Le père de l'écrivaine, directeur de la Marmorosh Bank à Bucarest, est mort quand sa fille avait neuf ans. Des relations tendues avec la mère incitent le lecteur à reconstituer les motivations, rébellions, décisions qui compensent les blessures d'une jeune fille construisant sa vie sans vrai appui dans un milieu ne favorisant pas la réalisation professionnelle des femmes. Les propos féminins rapportés montrent le talent journalistique de Maria Banus, ses dons de romancière (au ban de la société, elle écrit précisément un roman: "Les Vivants et les Morts»). Ils esquissent une typologie des comportements féminins négatifs sous le jour d'une fiabilité politique cruciale pour l'heure. Ainsi l'étourderie de Cici bavardant à propos de «NuȚa à présent dans l'illégalité $\rangle^{13}$ peut discréditer celle-ci au point de la faire arrêter. Que les femmes rencontrées soient ou non juives, le regard de la diariste maintient sa distance et son acuité. Loreta a un corps onduleux de Tsigane aux sens volcaniques, mais elle conduit d'une main de fer une entreprise textile prospérant avec la même efficacité que jadis les affaires de la grand-mère de Maria Banus. Ses options politiques inclinent-elles à soutenir l'extrême droite autochtone qui a passé un pacte avec Hitler en la personne du 
Maréchal Antonescu? Pour Loreta, le mal présent est attribuable au «peuple allemand coupable dans son entier qui doit être puni. Je soutiens qu'un peuple entier ne peut être coupable et châtié comme tel (...) Anton, têtu mène la discussion à mes côtés jusqu'à ses dernières conséquences. Soudain, il lance la bombe destinée à exploser sur ma cousine (...) : Loreta, tu penses comme une fasciste!...). Notre couple: Anton et moi - les brebis galeuses, communistes de la famille! »14 Marilena Drăgan, de même bord politique que le couple, n'inspire pas davantage confiance dans sa fierté de self-made-woman, son narcissisme, sa névrose.

Que de chemin parcouru depuis la petite fonctionnaire de 14 ans entretenant sa famille au statut de richarde d'aujourd'hui, épouse de grand homme d'affaires, sympathisante communiste. Il ne lui déplaît pas d'évoquer son passé. Elle prononce le nom de baptême du célèbre compositeur chef d'orchestre Alfred A. comme s'il était son intime. Je me souviens des snobs de Proust. - J'ai le socialisme dans le sang, me dit-elle. Ma mère aussi était socialiste. ${ }^{15}$

5 Les intellectuelles patentées rassurent-elles davantage? Le radicalisme anti-allemand de la Docteur S., son évaluation des culpabilités dans les crimes de guerre (les nouvelles d'horreurs perpétrées en Transnistrie affluent) font bon ménage avec son émancipation sous laquelle on peut soupçonner son goût des séducteurs, fascistes autochtones ou galants sans option politique manoeuvrant des frustrées.

Vieille fille, elle est camarade de faculté de Neli et de sa génération. Elle fait partie $\mathrm{du}$ petit contingent de femmes diplômées universitaires en Roumanie. Sa formation scientifique est solide, étroite, positiviste, avec quelque chose de sec, d'agressif de, sarcastique (...) Une ossature spirituelle de certitudes, de contentement de soi. Attention à ces vieilles demoiselles non sans coquetterie (...) Des cavaliers les courtisent, elles ont des liaisons discrètes dont elles disent : Un tel me fait la cour, Un tel me comble de fleurs et bonbons (...). Elle dit: Je suis contre les demimesures! L'ensemble du peuple allemand doit être punie pas seulement les chefs ! (...) Ils sont nombreux, c'est vrai : 80 millions ! Mais ce peuple doit disparaitre de la face de la terre $!^{16}$

6 Livia travaille en bénévole dans un hôpital militaire pour amputés telle son amie, l'actrice N.S. :

Livia a la pudeur de ses sentiments, elle n'aime pas faire cas de son labeur à l'hôpital (...). Elle est romancière, elle a traversé des crises mysticoreligieuses. Je lui dis pour blaguer: -(...) Tu consumes ton énergie, tu réalises tes aspirations de missionnaire et que tu disposes en plus d'un incroyable matériau de vie pour ta littérature ! ${ }^{17}$

7 Camelia monologue, parée d'un vêtement à boutons de métal ou de clochettes, enchantée d'elle-même, de sa lucidité, d'un rhumatisme infectieux qui lui procure en ces temps de tragédies collectives l'orgueil « d'une grande maladie, une plaie sociale telle les grands maux de naguère : tuberculose, syphilis... En ce moment, je suis en pleine crise, sens! J'ai de la fièvre... " De retour du restaurant Cina, elle raconte l'offense publique d'une amie envers une table voisine où dînent un homme célèbre et son épouse juive : «Regarde UN TEL et sa youpine (jidanca)!» " Moi, j'ai un esprit CONTEMPLATIF (...) Mon mari a fait une tête de six pieds de long, les autres idem! Moi seule suis restée impassible : je ne veux pas me fâcher avec elle! $»^{18}$

8 Si peu de réflexions sont émises par des femmes sur le genre dont elles relèvent, si nulle amie de confiance ne se montre dans l'espace confiné et ultra-fréquenté où la diariste n'entretient de relation proche qu'avec un mari montré comme un loyal camarade, des «théories sur les femmes» circulent dans la bouche d'hommes. Ils participent du tableau d'un extrême clivage idéologique et moral dans cette Capitale 
fasciste basculant dans le communisme à partir de l'Occupation soviétique en août 1944, vers la fin du Journal donc. Les communistes roumains étaient, dit-on, moins de mille à l'arrivée des Russes et la faiblesse numérique de cette résistance ne permet pas d'affirmer la sincérité de la « conversion » de 100000 personnes dès la pénétration militaire. Il est donc intéressant de voir le Journal commenter la transformation d'un légionnaire en communiste avant ou en cours de guerre. Pal décrit en ces termes, en avril 1943, le parcours de Costin, héros de l'Illégalité ayant dû vaincre sa misogynie.

Pal : - Savais-tu que Costin a naguère sympathisé avec les Légionnaires? Il ne s'en est pas éloigné pour motifs politiques, mais - comment te dire ? - » intellectuels » (...). Il était dégoûté, indigné par les gars de son foyer étudiant, des Légionnaires toujours saouls, faisant scandale : ils l'empêchaient de lire. Quand je l'ai connu, il s'en distançait. J'ai senti d'emblée le levain en lui, je l'ai pris dans mon secteur. Jusque tard dans la nuit, nous nous promenions ensemble sur la Chaussée. Ainsi a commencé sa formation communiste. Seigneur! Quelle puissance de travail! Supérieure à la mienne et même à celle des meilleurs ! Avant Pâques, je lui ai laissé une liste de livres à lire pour s'informer du principal (...). Les vacances finies, il les avait tous lus. Où se les était-il procurés? Mystère. Le comble, c'est qu'il les avait compris à fond (...). Quel élément exceptionnel! Hé bien si tu savais les préjugés qu'il a dû combattre! Sur les femmes ! (...) Il traversait des épreuves sentimentales et professait de ces théories, oh la la ! ...)- La femme animal de lit ? - Pas seulement ! - La femme, biologiquement inférieure ? - Si tu veux... Que de temps pour éliminer ces préjugés! Des moments politiques bien pénibles; ainsi quand il refusait qu'une femme lui soit supérieure dans la hiérarchie du Parti et puisse lui donner des ordres $^{19}$.

Ruth, mariée à Costin va être hébergée en fin de Journal. Condamnée à mort par contumace par un tribunal militaire, elle est cachée par le réseau de militants qui la soustraient à son sort. "Loger Ruth n'est pas plus dangereux que nos autres missions "techniques": servir de centre de réunions, cacher le matériel de propagande, recevoir et émettre des messages, donner des rendez-vous conspiratifs, rassembler fonds et vêtements pour détenus $»^{20}$. La condamnée expose d'elle-même les circonstances de son entrée dans le mouvement ("l'anecdote m'a horrifiée ») ${ }^{21}$. Adolescente, elle fréquentait des jeunes aux idées « avancées, incendaires " pour qui la femme est une « esclave exploitée par l'homme, la virginité n'étant qu'un aspect de l'esclavage. Pour échapper à ce stigmate de la servitude, elle a couché avec un garçon boutonneux qu'elle ne supportait pas $»^{22}$. L'anecdote recoupe l'atmosphère du roman de Mircea Eliade Noces au Paradis (1937) ${ }^{23}$. L'étudiante éperdue de lectures de romans russes qui en est l'héroïne, avant de devenir la femme fatale du roman, est d'abord présentée dans un salon bucarestois comme "la dernière vierge $d u$ $\mathrm{XX}^{e}$ siècle». Elle perdra cette virginité sans cérémonie, avec un officier qui la traite cavalièrement. Costin est en prison depuis trois ans et Ruth, qui l'aime, ne l'a encore jamais trompé quoique, dans l'Illégalité il l'ait souvent obligée à dormir dans le lit d'un camarade. L'un d'eux a tenté de provoquer un vilain scandale en l'accusant de préjugés petits-bourgeois. "Je ne sais si tout ce qu'elle me raconte est vrai. Je la crois préoccupée de faire mon éducation sexuelle révolutionnaire, puritaine et de paraître à mes yeux un modèle immaculé. Peut-être même est-ce son cas $»^{24}$.

\section{Nord des Balkans en guerre et Balkanisme de mœurs}

10 La guerre renvoie Maria Banus à une condition présentée comme antérieure à l'émancipation par le travail de sa grand-mère, antérieure à la vie de sa mère mariée bourgeoisement dans la Capitale, antérieure à ses propres réalisations individuelles, 
études universitaires et notoriété de femme de lettres au-dessus de la catégorie des cabotines amateurs. Elle la renvoie surtout à une régression. Âgée de trente ans en début du Journal, mariée, elle s'interroge à plusieurs reprises non sur son aptitude, mais sur son droit à concevoir un enfant, situation peu envisageable en termes de choix quand le militantisme communiste commencé en 1937 implique le qui-vive et et le risque continuel d'une arrestation : "nos godillots de montagne, à Anton et à moi, bourrés de chaussettes de laine, graissés à la graisse de poisson, ressemelés de frais attendent dans le placard depuis un an une éventuelle déportation $»^{25}$. On note par ailleurs - information non communiquée dans le Journal - que Maria Banus a été portée en 1941 sur la liste de 42 écrivains déclarés ostateci/ otages par le régime Antonescu. Avec Sarina Pas Casvan (écrivain pour enfants et traductrice) elle était la seule femme de cette affiche de novembre 1941 placardée dans les lieux publics sous le titre «Scriitori evrei/ Écrivains juifs » mentionnant date, lieu, nom de naissance, nom des parents, pseudonymes, titres des livres publiés. La publication et la vente de livres de tels auteurs étaient interdites et l'affiche stipulait qu'ils seraient retirés des bibliothèques scolaires et publiques. La liste a inclus des noms d'écrivains décédés ou émigrés de Roumanie (ainsi Benjamin Fondane, Tristan Tzara, Ilarie Voronca). Par ailleurs, leur statut a été personnellement communiqué aux auteurs (vivants en Roumanie en tout cas) qui, quelques jours après l'affichage, ont été convoqués à la Préfecture de Police où on leur a communiqué que déclarés otages, "ils paieraient de leur tête si "quelque chose" se produisait / dacă se va îtâmpla 'ceva' ei vor plăti cu capul »".

11 L'inaction est compensée par d'incessantes lectures, Hemingway (juin 1944) ${ }^{27}$, Malraux (29 novembre 1943) «je relis La condition humaine ${ }^{28} »$, Platon (19 mai 1943), «J'ai lu avec Hari l'Apologie et Criton - Le divin Platon! Dit Hari. ${ }^{29}$ ", Hemingway encore (15 août 1943) "J'ai lu un livre extraordinaire Pour qui sonne le glas de Ernest Hemingway. Il parle de l'Espagne pendant la guerre civile $»^{30}$, Huxley (12 août 1943) ${ }^{31}$, Mircea Eliade (12 août 1943) "Les Hooligans : j'ai cherché ce livre chez des bouquinistes, je l'ai trouvé. J'étais très curieuse de le lire. Adrian m'en avait parlé en me disant que "l'autre camp" y est assez bien décrit. Le problème de la jeune génération d'intellectuels désespérés, courageux, cyniques, sincères envers eux-mêmes jusqu'à l'auto-flagellation est clairement souligné par Eliade. Mais le seul personnage pourvu d'une véritable vie me semble David Dragu (qui met en scène Emil Cioran?). Les autres héros représentent tout juste des catégories sociales, des idées. On les a fabriqués habilement, mais ils ne sont pas viables. ${ }^{32}$-, Miron Radu Paraschivescu (16 août 1943) - "Cîntice Tigănești/ Chants tsiganes». "Je montre à Hari ce livre qui me plait à la folie. »" Des lectures se font en allemand. Ainsi Fritz Künkel (27 mars 1943) « Einführung in die Karakterkunde» dont elle lit des fragments à Anton ${ }^{34}$. Des livres anglais sont consommés aussi. «Livres lus ces derniers temps: Clare Sheridan "Nuda Veritas», Dickens "Le mystère d'Edwin Drood ", "Les aventures de Mr. Pickwick», "Vies et aventures de Martin Chuzzlewit », Mircea Eliade "s sarpele/ Le Serpent "35 ( $1^{\mathrm{er}}$ avril 1943). Des sorties sont possibles, une matinée au théâtre permet d'entendre "Don Carlos» de Schiller (« Mise en scène faible, acteurs médiocres » 18 avril 1943) ${ }^{36}$.

12 Le nombre des activités interdites aux Juifs augmente et Sous Camouflage décrit au fil des rencontres le piège qui se resserre sur cette population désormais impossible à confondre avec nulle marge sociale ou pénale du pays, si bas placées que soient ces dernières, hormis l'autre catégorie ethnique concernée par la déportation en Transnistrie, les Tsiganes. S'informer sur la progression réelle des opérations de guerre est impossible comme le lecteur le constate quand les protagonistes collent l'oreille à la 
paroi murale d'un appartement voisin écoutant Radio Londres. "Nous n'avons pas le droit d'être propriétaires, d'avoir des usines à notre nom, d'exercer des professions libérales (j'ai été radiée du Barreau), d'engager du personnel de service, de posséder des automobiles, de disposer du téléphone, de machines à écrire. Mais c'est l'absence de radio que nous ressentons avec le plus

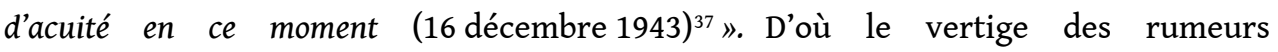
fantasmatiques, sur la Transnistrie, la démission en Italie de Mussolini apprise par un quidam sur Radio Londres, répétée avec retard, notée le 27 juillet 1943, alors que l'événement est déjà fêté en privé. La défection entraînerait l'abrogation des lois raciales, ce qui réjouit les Juifs mais aussi le colonel B. par exemple : "En définitive, quelle bonne nouvelle pour les Roumains! C'est bien Mussolini qui a dit que nous étions tsiganes, que nous servions d'esclaves aux Romains et qui nous a pris la Transylvanie. Bien fait pour lui ! $\aleph^{38}$. Les bruits sur les opérations de guerre se nourrissent de réflexions sur la culpabilité comparée des fascistes roumains et des Allemands («Lea pense à Nica, fusillé lors de la rébellion -légionnaire- de 1940 avant d'être pendu à un crochet de boucherie à l'abattoir " ${ }^{39}$. Ils se tempèrent d'humour à l'occasion d'épisodes drôlatiques complaisants, telle une visite de S.S à domicile. Quand on sonne à une heure tardive Anton se trouve dans la salle de bains, la mère de Maria et Ruth, l'hôte condamnée à mort par contumace, dans le hall. "Il fait noir dans le vestibule. Nous n'allumons pas la lumière. Dans le champ visuel de "l'œill" apparait la partie centrale d'une silhouette et un insigne : tête de mort et ossements croisés. En une seconde, l'agonie, la glace. Tout tremble. Ouvrons! Dit Anton $»^{40}$. Un des S.S est I. K, chef de chantier du mari de Maria Banus à l'époque où ce dernier était ingénieur à Brasov. Son camarade, paysan souabe des environs de Timișoara porte $u^{41}$ uniforme aussi avachi que le sien car tous deux sont épuisés par une longue route.

La maîtresse de maison met la table, apporte de la viande froide, Messieurs les sousofficiers s'excusent de déranger, pas du tout, ça fait longtemps qu'on ne s'était vus, vous prendrez bien un peu de liqueur de cerises, si seulement cette saleté de guerre prenait fin! Le camp ${ }^{42}$ de Oranienbourg c'est à devenir fou quand on est posté dans la tour de guet... Où est passée ma mère? Une crise cardiaque sur le divan? Continuons la conversation. Les détenus électrocutés sur les barbelés quand ils tentent de fuir. Des dingues! On dirait qu'ils cherchent la mort... - Pas nous! dit le Souabe en homme de bon conseil. C'est elle qui nous court après. Dans quelques mois, écoutez-moi bien, Monsieur l'Ingénieur, tous ces réverbères que vous voyez en bas sur le boulevard, ils porteront des pendus de notre sorte ${ }^{43}$. (mercredi 8 décembre 1943)

Les S.S sont venus proposer à la vente un lot de semelles dont nul n'a usage dans les camps, les détenus portant des sabots de bois. "Si Monsieur l'ingénieur pouvait placer ne serait-ce qu'une petite partie de la marchandise? $»^{44}$. Après des vœux réciproques, «Je me sens fourbue comme si j'avais escaladé l'Everest». On ne saurait mieux montrer la corruption et la corruptibilité de S.S. locaux psychologiquement typés en Roumains qu'ils redeviendront après l'intermède fasciste/ nazi à la glorification ou instrumentalisation de leur groupe.

Faible se révèle aussi la fiabilité des camarades de clandestinité chez qui sentir les signes de relâchement de solidarité de Parti, en coïncidence avec l'obsession antisémite la plus évoquée, la passion des juifs pour l'argent. Mitran âgé de 40 ans, plus gradé qu'Anton dans la hiérarchie du Parti, à peine libéré de camp de concentration (lagăr), sans doute promis à une fonction de ministre en cas de victoire communiste, tient à son tour des propos sur la corruption (Mitran a feuilleté sur les rayonnages de ses hôtes un ouvrage français de Ch. Guignebert Le monde juif au temps de Jésus : «Laissez tomber, les 
Juifs sont en faute. Ils graissent la patte à droite, à gauche, ils méritent leur sort. (...) - Ainsi commence l'antisémitisme. - La tendance des juifs à se sauver par l'argent s'explique par l'histoire. Une population habituée à distribuer des pots de vin depuis des milliers d'années !... »" ${ }^{45}$ (27 juillet 1943). Comme les autres militants, Mitran est froid, retenu (12 août 1943). Sans doute, tous les communistes se montrent-ils circonspects, prévoyants, mais cet homme puissant réagit avec une froideur délibérée pour refuser l'entretien que Maria Banus lui réclame. "Il se tait. Son expression reste inchangée. Son visage massif, ses yeux clairs tiennent de l'idole orientale: pierre et verre. Je me tais à mon tour $»^{46}$.

15 Face à Mitran, à Anton, à Dionisie, à Hari, à Jac, les principaux hommes du livre, l'impuissance se combine à des fantasmes de culpabilité délirants. Quand Jac, compagnon de sa mère venue vivre sous leur toit bénéficie d'un diagnostic médical pour sa maladie "honteuse", syphilis non traitée de longue date, la narratrice est frappée par "une immense nausée, un vide dans l'estomac " ${ }^{47}$. Le malaise renvoie à une double tare sexuelle et sociale : l'adéquation de la diariste, de sa mère à une image mythologique maléfique de la femme dont participe aussi la séductrice qui a contaminé Jac et l'obséquiosité ou l'impuissance du petit employé israélite désigné par ailleurs dans le Journal comme germanophile.

Au-dessus de notre maison plane la Femme-Araignée, nue dans ses longs bas, sexe noir, étoile du Caf'Conc' de Galatzi fin de siècle. Caviar noir, champagne! Qu'il marchait au pas, empesé, manchettes immaculées, le doux fonctionnaire de l'entreprise FischerGalati menu et snob, flatté par l'attention du fils de son patron: Heinrich, un type raffiné ayant fait ses études à Vienne, érotologue, bibliophile. Quelle fierté d'être autorisé à le suivre au Café-Concert, dans sa société d'intellectuels de bonne naissance ${ }^{48}$.

Divers développements sur la mystique, les profanations du désir amoureux se formulent comme un contre-pied de la moralisation fasciste au pouvoir. Non exempts d'un malaise qui se communique à la lecture, on les attribue à une velléité de chronique de l'avilissement des femmes par la violence, l'utilisation érotique, le voyeurisme pouvant expliquer la censure communiste ultérieure sur la période. Ils sont à rapporter sans doute aux rumeurs réelles ou fabulées sur la Transnistrie (adolescentes violées puis abattues par les S.S avant la visite d'épouses constatant que leurs maris ont contrevenu aux lois raciales, détenues triant nues le maïs dans des hangars en plein vent par plusieurs degrés sous zéro etc...). Ils se relient aussi à des fantasmes présentés comme individuels sans doute exacerbés par la défiance, l'impénétrabilité croissante des militants rencontrés. Le contour de ces fantasmes est si nettement dessiné, ils apparaissent comme si compensatoires de la faiblesse/ solitude morale et affective de la femme-artiste qu'une lecture attentive pourrait les prendre pour une élaboration semiartistique, romanesque, de l'ordre de celle qui préside peut-être à l'écriture de son roman, Les Vivants et les morts. Ainsi, mariée à Anton, la protagoniste continue d'éprouver pour Dionisie - amour de jeunesse marié et père, militant incertain - une passion désespérée nourrie du dédain de cet homme, des souvenirs d'une adolescence révolue dans une Capitale dont nombre d'écrivains, metteurs en scène, chanteurs, compositeurs ont célébré avant-guerre la sensualité ambiante, aujourd'hui confondue avec la facilité poétique de naguère.

Aujourd'hui vendredi, sur les quais de la Dâmbovitsa. Soleil et vent. Il est si rare que je puisse m'enfoncer dans la nature, dans la profondeur de l'existence jusqu'à la douleur 
de l'enfant, de l'adolescente, de la poétesse. Sans doute je n'aime pas assez, je ne me donne pas assez, je ne rêve pas assez. Mon désir insatisfait se transforme en aigreur de vieille ${ }^{49}$.

Cette passion pour un homme qui se dérobe (il n'est pas venu à un rendez-vous relevant confusément de l'urgence érotique et politique à la fois) est si pénible, frôle si bien la trahison idéologique que la narratrice la confie à son mari, exigeant de communiquer à Mitran un détail qui la torture : elle a accepté de l'argent de Dionisie (alors que sa "réputation dans le Mouvement est mauvaise»). Crainte ou désir de voir un Dionisie s'impliquer à son tour dans un réseau de corruption? Fantasme de prostitution tel celui qui pèse sur la maison depuis la révélation de la maladie de Jac? «- Oui, Tu as commis une faute politique, dit Mitran. Et il se tait. $"^{50}$ (12 août 1943). Après l'un des bombardements de Bucarest concluant le Journal, la diariste incarnant "le gastéropode suprasexuel, supramusical » de la poésie de Ion Barbu, va fantasmer une mort des amants transgressant symboliquement les tabous de leurs histoires culturelles incompatibles: "La nuit, je rêve d'une mort wagnérienne (...) Enfoncés dans une toile musicale qui nous enveloppe tel un suaire, Dionisie et moi formons un tout qui disparaît dans un néant euphorique. " "119 mars 1944)

Hari l'ami, sans doute d'enfance, rencontré presque chaque jour, qui partage avec elle ses lectures philosophiques, des commentaires de théories marxistes, l'assomme de confidences et du récit de ses lectures et travaux (théoricien acharné, il se projette dans le futur politique où il réalisera l'œuvre conforme à ses aspirations à la fois tourmentées et simplistes). Dans un mélange de compassion et de distance, il est montré comme un raté. De même structure mentale, sociale que les élèves d'extrême droite de Nae Ionescu, étudiants en philosophie sans travail bâtissant en pensées et intrigues un avenir fasciste proche de la Cité socialiste idéale d'Hari, il partage pourtant avec elle la conviction de relever d'une catégorie humaine marquée d'infamie. Une de ses amantes s'est mariée dans le seul but de combattre l'attraction qu'il exerce (elle a "des goûts érotiques spéciaux ») ${ }^{52}$. Si des disputes continuelles jettent l'un contre l'autre Hari et Maria Banus, des moments de paix les unissent. Ainsi la discussion d'une caractérologie sur la séparation, mais aussi la complémentarité des types psychologiques pouvant se surimposer à l'ancienne distinction entre créativité masculine et féminine, dont la protagoniste développera les prémisses culturellement acceptables, dans le cadre du foyer de culture juive autorisé dans la Capitale depuis 1941, le Baraseum.

21 Le type politique, d'action, vit éthiquement, politiquement, en permanence les problèmes de son temps. Le type du penseur, de l'artiste a ses problèmes, ses difficultés (...) La philosophie du matérialisme dialectique doit être créée. Comprends-tu notre responsabilité ? Si nous nous contentions de simple exégèse et du commentaire « des grands » nous ferions œuvre d'épigones décadents, alors que dans une culture jeune, en formation... Et toi qui as la chance de prendre part à une telle œuvre de création d'une culture, tu gémis, tu bougonnes, tu cherches la petite bête... Je pleure un peu, radoucie. Comme elles sont rares nos heures d'amitié sereine, de calme discussion !53 (26 novembre 1943)

22 Les rêves nocturnes et leur décryptage (reçus et transcrits pour leur richesse, ils sont envisageables comme une preuve du débordement de la créativité, forcée au 
refoulement par l'impossibilité de publier ou d'agir) jouent un rôle, suggérant d'une part :

- une communauté d'inspiration de Maria Banus avec le dadaïsme début de siècle de Tristan Tzara et le surréalisme bucarestois d'après guerre (Paul Celan Paul Păun avec lequel elle a d'ailleurs entretenu dans les années 1930 une correspondance) dont les femmes créatrices se révèlent absentes.

- une éventuelle influence du freudisme. Inconcevable dans cet ouvrage publié sous communisme (la psychanalyse y était interdite), ce courant n'est jamais mentionné. Mais on pourrait trouver sous l'invocation ci-contre entre éveil et rêve une éventuelle esquisse des trois étages de la structuration freudienne de la personnalité, -ça, moi, sur-moi-, coïncidant chacune avec une forme de l'accord avec les trois hommes du livre dont chacun incarnerait une facette de la Destinée de guerre de la femme qui écrit. En l'absence de toute possibilité de projet personnel, Dionisie, Anton, Hari figuent aussi les repères à partir desquels le désir de vivre, la confiance en soi, en ses propres pouvoirs libidinaux se structure, chacun de ces hommes jouant un rôle dans l'impression bancale d'équilibre, de sécurité, de possibilité de survie.

Rêve de Cabbale, rêve crépusculaire, yeux grands ouverts. Je parlais à Dionisie : j'ai trois âmes, l'une dans le ventre, l'autre dans le cœur, la troisième dans la tête. Tu as trois âmes aussi. Les âmes de nos ventres s'aiment. Ton âme du cœur aime ton enfant, rien que lui. Mon âme du cœur s'appuie sur Anton. Nos âmes du ventre se contorsionnent, s'appellent, se cherchent. Nos têtes sont ennemies. La tienne est une bête sauvage. Elle ne connaît aucune loi morale. Elle ne brûle que pour l'argent et le pouvoir. Dans ma tête se sont allumées des étoiles qui me tourmentent. Et tu te moques d'elles, de ma naïveté. L'âme de mon ventre est tienne. Nous ne faisons qu'un. (mercredi 8 décembre 1943)

\section{Figures de chrétiens orthodoxes et de juifs}

En 1943, la diariste souligne son enracinement (Je fais partie de ce qu'on nomme une famille assimilée. Ma grand-mère parle une belle langue valaque, des bords de la Dâmbovitsa (...). Tous les miens ont été des indifférents religieux. De la vieille matrice judaïque, ils n'ont jamais conservé qu'une sensibilité aiguë marquée de scepticisme et d'humour " ${ }^{54}$. L'appartenance roumaine est donnée comme acceptée, désirée même, quoique refusée par autrui. Pour caractériser son désir déçu de fusion identitaire, le «je » du texte use des termes comme "bovarysme ethnique roumain" où nous voyons une allusion à la théorie philosophique de Jules de Gaultier, d'après l'idée du roman de Flaubert. Ce bovarysme (désir de se conformer à une identité à construire par la volonté, l'activité) tend à représenter comme un foyer paradisiaque "l'alvéole mioritique » roumaine. Soit l'espace idéal défini alors par le philosophe L. Blaga d'après la ballade pastorale Miorita - où un berger fabule sa mort mystique sur un plateau coin de paradis face à deux rivaux préméditant son assassinat. Face à cette construction mentale, la diariste affirme avoir ressenti avec acuité "le sentiment de marge " ${ }^{55}$ qui est sien. C'est pourtant une scène vécue à la piscine, aux risques et périls de la protagoniste puisque l'accès d'un tel lieu est interdit, qui procure l'une des plus fortes impressions de rejet, de scandale moral. Le bassin et son pourtour constitue en effet un îlot de luxe sportif, mondain, où s'ébat dans l'insouciance une jeunesse affichant son droit aux privilèges et l'identité religieuse qui les autorise. 
L'eau est bleue, cristalline, les éphèbes bronzés portent des croix d'argent sur leurs poitrines. Les femmes au corps verni (...) au-delà du bien et du mal se dorent au soleil. Les soutiens-gorge décolletés : au-dessus de petites croix d'or (...). Il n'y a pas de guerre. Le temps n'existe pas. Les jeunes corps sont détendus dans la lumière. Sentiment de culpabilité, de gêne. Qu'est-ce que je fais parmi ces gens? J'ai nagé. Mes muscles se sont réveillés avec bonheur. Puis l'idée que j'étais coupable m'a frappée, que je n'avais pas le droit de me sentir si bien (...) et j'ai éprouvé du dégoût pour moi-même tout en craignant qu'une inspection me démasque. Je n'ai pas le droit de prendre de tels risques alors que j'ai chez moi du matériel de Parti et que tout contrôle mineur peut entraîner une perquisition ${ }^{56}$.

S'affirmant dépourvue de sentiment d'appartenance judaïque initial (autre que des préférences alimentaires, le goût d'expressions verbales), découvrant dans «le gris vacuum de son indifférence religieuse un rationalisme de type illuministe ou un messianisme de type révolutionnaire ${ }^{57}$, la diariste suggère qu'elle aurait pu être portée vers le nationalisme orthodoxe chrétien des années 1930 si le positionnement antisémite de ce dernier ne l'avait d'emblée exclue.

Quand j'avais vingt ans et que la vague du mysticisme nationaliste orthodoxe grandissait en Roumanie, j'ai envié ceux qui se laissaient soulever par ce qui semblait une rédemption. J'étais abandonnée sur le rivage, seule, à l'écart, à écouter la tempête. De son mugissement obscur montaient des cris contre une collectivité qui était mienne sans l'être, mais m'englobait ( $\left.{ }^{58} . ..\right)$ Pas jusqu'à l'identification pourtant. Quand j'entends l'idiome venu d'Allemagne (le yiddish) passé par les ghettos polonais et russes, modulé, chanté, traînant, une vague de sang brûlant m'inonde le visage. J'ai honte de ma répulsion face à la vieille langue de la diaspora et parfois face à ceux qui la parlent : des faces pâles, enlaidies, rendues grimaçantes par des siècles d'humiliation et de misère. Toujours les victimes sont laides, inesthétiques. Mais sans doute ma répulsion est-elle d'une autre nature que celle des non-juifs... ${ }^{59}$

La fréquentation du Baraseum, signalée en août 1943, va proposer une issue au sentiment de marasme découlant de l'inaction forcée, à l'emmurement politique, aux obsessions fantasmatiques proches de la dissociation psychologique. Il renvoie à une réalité de la vie artistique roumaine relativement connue et commentée. Autorisée en 1941 à la suite d'un « compromis » autorisant les artistes juifs à se produire dans ce seul cadre et exclusivement en roumain ${ }^{60}$, l'existence du Baraseum a pu être utilisée comme preuve de tolérance du régime fasciste d'Antonescu face à la culture juive ${ }^{61}$. Si le point de vue n'est pas entériné sous cet angle par Sub camuflaj, il fait cependant état d'une rupture dans la logique narrative du Journal. À partir de cette première invitation au théâtre juif s'élaborent des contacts, des collaborations grâce auxquelles les projets de l'artiste et de la femme se reconstruisent à toute allure, loin des activités militantes mentionnées jusque-là, mais aussi de la méditation sur les antinomies de la création artistique (...) ${ }^{62}$. La soirée littéraire signant le début d'une reconstruction de soi sous le signe de la reconnaissance d'une fonction sociale de l'artiste est organisée par des écrivains de langue roumaine, mais des auteurs yiddish sont présents. Sous-tendue par l'interdiction d'expression dans cette langue à partir de 1940, la manifestation est animée de petites scènes esquissées surtout dans la deuxième partie du spectacle qu'organisent des yiddishisants. Des traductions confrontent la poétesse à son manque de familiarité avec un milieu qui est aussi hébraïsant, sioniste, etc. On s'adresse à elle avec une curiosité, une admiration attestant qu'on se souvient au moins de sa notoriété d'avant-guerre, que sa réputation dans ce milieu s'est élaborée dans un soutien fervent.

Vous êtes Maria Banus? Savez-vous que je vous ai traduite? Que des vers de vous ont paru en Palestine? - Qu'avez-vous traduit? - Je ne sais plus, je dois chercher. 
J'ai traduit près de 400 poèmes du roumain! À ma droite : Enric Furtuna. - Tu entends, Furtuna, la question de madame B. ? Si je sais l'hébreu? Madame, on m'a décerné le prix Bialik. Sans parler d'un autre prix que j'ai reçu à Moscou. Je suis gênée par mon manque d'information en matière de poésie yiddish et hébraïque. J'en sais sans doute davantage sur la poésie chinoise, japonaise... ${ }^{63}$

L'invitation est suivie de plusieurs autres donnant à connaître des actrices dont l'une chassée du théâtre où elle travaillait, mais aussi Sara Manu, la plus jeune actrice du Baraseum dont l'aura va se confondre en esprit avec celui de Ruth comme en réparation de l'avilissement présent d'un groupe ethnique et d'un genre: «un elfe possédé de visions. Après la guerre, quand sa flamme pourra se déployer librement, je suis sûre qu'elle deviendra une gloire du théâtre roumain $»^{64}$. Un compositeur rencontré à une soiréeconcert, Alfred Mendelssohn, veut composer un accompagnement à un poème qu'elle souhaite mettre en scène. "Je me sens ivre, exaltée à l'idée que ma poésie puisse dire quelque chose à des humains..$^{65}$ " Le compositeur "blond, grassouillet, au rire facile » et elle-même travaillent effectivement à un motif musical sur le thème du "Bucarest de l'enfance » et envisagent des réalisations dont la plus signifiante dans le contexte du Journal est le traitement artistique d'épisodes de la Shoah roumaine dont leur collectivité est en train de vivre l'expérience.

Je demande à Mendelssohn : ne crois-tu pas que l'exode de Transnistrie pourrait être à la base d'un oratorio profane ? Ce thème m'obsède et pèse sur le coeur de beaucoup. Mais sans doute dans le futur n'intéressera-t-il pas, ne se trouvera-t-il pas dans la ligne de l'art? Pourquoi? S'étonne-t-il. L'art ne changera pas tant que ça. La tragédie d'une population qui a donné un si grand nombre de victimes trouvera un écho dans toute société nouvelle ${ }^{66}$. (mercredi 8 décembre 1943)

Par ce dernier aspect surtout, ce Journal publié en 1977 nous paraît présenter un intéressant point de comparaison pour les révélations esthétiques apportées par Paul Celan, par Norman Manea, prix Médicis étranger 2006. Ces deux auteurs ont partiellement élaboré leur œuvre littéraire à partir d'une représentation du traumatisme hitlérien personnellement vécu. P. Celan, comme N. Manea, ont tous deux avant leur reconnaissance en Occident payé le prix fort de la non-reconnaissance artistique au nom d'arguments négationnistes de la Shoah en Transnistrie, mais aussi en raison de moralisations déclarant déplacée (à la suite d'Adorno) la création de poésie après Auchswitz. Ni P.Celan, ni N. Manea, ni Mihail Sebastian, auteur d'un Journal 1935-1944 dont la traduction française par A. Paruit a rencontré le succès, ne peuvent être taxés de collaboration avec un communisme roumain institutionnalisé, puis la dictature de Ceaucescu. Celan a quitté la Roumanie en 1947, N. Manea était enfant lors de son retour de déportation, M. Sebastian est mort en 1945, écrasé accidentellement par un camion à Bucarest. Aucun de ces écrivains n'a pu se trouver en situation de conduire un projet artistique composant avec des directives de Parti par exemple sur des réalités sciemment occultées de la Seconde Guerre mondiale. Le succès de Maria Banus est susceptible d'avoir procédé de l'opportunisme, mais aussi de la cynique utilisation par le régime d'un talent et d'un statut sans issue, tant à des fins de détournement de l'histoire roumaine juive que de l'exploitation d'affects féminins. C'est à ce double titre que sa poésie, dont son Journal 1943-44 a prétendu aussi refléter la face cachée nous paraît devoir être réétudiée. 


\section{BIBLIOGRAPHIE}

Arendt Hannah, (1974) Vies politiques (H. Arendt : 1955, 1960, 1965, 1966, 1967, 1968, 1971), Textes traduits de l'anglais et de l'allemand par Éric Adda, Jacques Bontemps, Barbara Cassin, Didier Don, Albert Kohn, Patrick Lévy, Agnès Oppenheimer-Faure. Paris : Gallimard, 1974 pour la traduction française.

Banus Maria, (1977), Sub camuflaj. Jurnal 1943-1944, Bucarest : Carte românească.

Bensoussan Georges, (2011), «L'horreur oubliée : la Shoah roumaine ». Revue d'histoire de la Shoah, $\mathrm{n}^{\circ}$ 194. Paris : Revue d'Histoire de Documentation juive, janvier-juin.

Mirodan Alexandru, (1986), Dictionar neconvenȚional al scriitorilor evrei de limba română (ABC), Tel Aviv :Editions Minimum.

Negrici Eugen, (1999), Literature and Propaganda in comunist România, Bucharest: The Romanian Cultural Foundation Publishing House.

Neumann Victor, (1996), Istoria evreilor din România ( Studii documentare și teoretice).

Timișoara : Editura Amarcord.

Sebastian Mihail, (1996/2007), Journal 1935-1944. Traduit du roumain par Alain Paruit. Paris : La Cosmopolite, Stock.

\section{NOTES}

1. Ainsi Géo Serban, O. Morar soulignent en accord avec les fils de la poétesse l'intérêt de restituer l'intégralité du Journal commencé à l'âge de 14 ans, poursuivi jusqu'à son décès en 1999 et proposent d'établir une édition rigoureuse des manuscrits.

2. Voir l'article en roumain "Societatea Scriitorilor români», Wikipédia. Page consultée le 27 août 2012.

3. Voir Maria Banus, Wikipédia. Page consultée le 27 août 2012.

4. Voir Wikipédia "Résistance en Roumanie pendant la Seconde Guerre mondiale ». Dernière modification : 29 août 2012. Page consultée le 30 août 2012. Ce Conseil national de la Résistance a tenté de négocier en Suède, selon Le Monde du 25 août 1944, (« Un jour pour se retourner ») un «changement d'alliance au profit des Alliés occidentaux» et il a demandé un débarquement anglo-américain dans les Balkans. Les négociations ont été conduites (en Suède) par Frédéric Nanu et son agent Neagu Djuvara, qui deviendra historien, et, en Turquie, par le prince Barbu știrbey.

5. 19780 selon notre exemplaire.

6. Cité par A. Mirodan dans Dictionar neconventional al scriitorilor evrei de limba română p. 267, d'après Maria Banus "Mit, realitate și etern feminin', fragmente, dans le volume 'Himera', București, 1980, p. 230-231.

7. Journal, p. 16.

8. Ibidem, p. 16.

9. Ibid., p. 17.

10. Ibid., p. 45-46.

11. Ibid., p. 94-95.

12. Ibid., p. 55, 56, 59, 60.

13. Ibid., p. 48-49. 
14. Ibid., p. 14.

15. Ibid., p. 12.

16. Ibid., p. 195-196, 31 août 1943.

17. Ibid., p. 28-29.

18. Ibid., p. 88, 23 avril 1943.

19. Ibid., p. 88-89, 23 avril 1943.

20. Ibid., p. 315, mercredi 8 décembre 1943.

21. Ibid., p. 323 mercredi 8 décembre 1943.

22. Ibid., p. 323.

23. M. Eliade. « Nunta în cer/ Noces au paradis». Traduit par Marcel Ferrand, L'Herne. 1981.

24. Ibid., p. 323.

25. Journal, p. 338, mercredi 8 décembre 1943.

26. Voir Wikipédia. «Scriitori evrei ostateci», dernière modification de la page : $1^{\mathrm{er}}$ mars 2012. Page consultée le 31 août 2012.

27. Journal. « Le soleil se lève aussi » mentionné p. 403.

28. Ibid., p. 305.

29. Ibid., p. 113.

30. Ibid., p. 119.

31. Ibid., « Point contrepoint » mentionné p. 151.

32. Ibid., p. 152.

33. Ibid., p. 165.

34. Ibid., p. 30.

35. Ibid., p. 47.

36. Ibid., p. 72.

37. Ibid., p. 340.

38. Ibid., p. 125-126.

39. Ibid., p. 127.

40. Ibid., p. 337, mercredi 8 décembre 1943.

41. Ibid., p. 337.

42. Ibid., p. 337.

43. Ibid., p. 337.

44. Ibid., p. 338.

45. Ibid., p. 133-134.

46. Ibid., 12 août 1943, p. 149.

47. Ibid., 18 avril 1943, p. 71.

48. Ibid., p. 71.

49. Ibid., p. 299. 26 novembre 1943.

50. Ibid., p. 149. 12 août 1943.

51. Ibid., p. 366.

52. Ibid., p. 290, 17 novembre 1943.

53. Ibid., p. 292.

54. Ibid., p. 183, 19 août 1943.

55. Ibid., p. 183.

56. Ibid., p. 128-129, 27 juillet 1943.

57. Ibid., p. 183.

58. Ibid., p. 183-184, 23 août 1943.

59. Ibid., p. 184.

60. Voir Clotilde Goujart Le théâtre yiddish en Roumanie, héritage d'un patrimoine commun. Page du 01/07/2011 consultée le 31 août 2012. http://www.regard-est.com/home/breve_contenu.php? id $=1217$ 
61. L'article de Wikipédia sur Radu Gyr (1905-1975), commandant légionnaire et directeur des théâtres sous le régime Antonescu (septembre 1940-janvier 1941) souligne que l'existence d'un théâtre juif dans une capitale fascisée a été commentée sur le plan international à de nombreuses reprises avant 1989. Radu Gyr, Wikipédia, dernière modification : 2 juillet 2012. Page consultée le 31 août 2012.

62. Journal, p. 327-328, 8 décembre 1943.

63. Ibidem, p. 186, 23 août 1943.

64. Ibid., p. 320, 3 décembre 1943.

65. Ibid., p. 320, 3 décembre 1943.

66. Ibid., p. 329-331.

\section{RÉSUMÉS}

L'analyse présente Sub Camuflaj/ Sous camouflage publié à Bucarest en 1977. Il s'agit du journal de fin de guerre de la poétesse Maria Banus (1914-1999) auteur aussi du célèbre recueil : Tara fetelor/ Le pays des jeunes filles, 1937. Pour le critique israélien A. Mirodan, le livre aurait fait scandale par son immoralité sexuelle (relation avec des hommes sous le toit conjugal, évocations sarcastiques de compatriotes menacés de déportations peu connues en Occident - camps de Transnistrie -). L'auteur de Sous camouflage a été largement publiée et traduite pendant la période communiste. Opérant en 1943-44 son parcours, dans l'Illégalité, elle n'en décrit pas moins le clivage entre Juifs de son entourage animés par l'espoir d'un renouveau lié à l'arrivée soviétique et jeunes Roumains installés dans le camp d'une droite nationaliste chrétienne orthodoxe imposant au quotidien ses symboles sinon sa loi dans la capitale. Plusieurs détails présentent ce groupe comme plus légitime que le premier. Comblée de récompenses après guerre, $\mathrm{M}$. Banus tombe dans l'oubli dans les années-80. Réfugiée en France, elle fait figure d'opposante à la dictature Ceaucescu. En 2008, son œuvre n'était toujours pas sortie d'un purgatoire attribuable selon le jeune critique O. Moraru autant à l'antisémitisme qu'à la misogynie. De fait, Sub camuflaj réunit les qualités reconnues à M. B. au temps de sa gloire. Il est journal de création (avocate radiée du barreau, elle écrit un roman), chronique de rues, préparation mentale à l'arrestation, mais aussi saga familiale (souvenirs d'une lignée juive assimilée modelée par la personnalité d'une grand-mère fournisseur de personnalités proches de la Maison royale). On est tenté de comparer ce texte au Journal 1935-1944 de Mihail Sebastian publié en français en 1996 (meilleure vente de traduction roumaine après la chute du Mur de Berlin). Mihail Sebastian aussi a été marginalisé comme juif pendant la guerre, mais son oeuvre littéraire loin de tomber dans l'oubli, connaît aujourd'hui maintes rééditions.

This analysis presents Sub camuflaj: 1943-44 / under disguise: 1943-44 published in Bucharest in 1977. It is the diary of the war's end by Maria Banus (1914 - 1999), who is also the author of Tara fetelor / the maiden country. For the Israeli literary critic A. Mirodan this diary was scandalous due to its sexual immorality (relations with men in the matrimonial home) and due to sarcastic references to compatriots then threatened by deportation (Transnistria camps). The author of Sub camuflaj was widely published and translated during the communist period. Working Illegality in 1943-44 she describes the cleavage between jews excited by the hope for a renewal linked to the soviet arrival and young Romanians belonging to a nationalist, christian orthodox right, while the "legionaries" daily imposed their symbols if not the law in the capital. Several details show this 
group to be more legitimate and desirable than the first one. Heaped with praise after the war, $\mathrm{M}$. Banus is forgotten in the eighties. As a refugee in France she opposes the Ceaucescu dictatorship. In 2008 her works remained unknown, a fact that the young literary critic O. Moraru attributes as well to antisemitism as to misogyny. Actually Sub camuflaj unites the qualities attributed to M. Banus durig the era of her glory: it is a diary of creation, a chronicle, a conjuration of daily fear, but also a saganovel. Its diarist intention avoids as well nationalist or religious as dogmatic feminist speech. One can compare Sub camuflaj to Journal 1935 - 1944 by Mihail Sebastian -1998 (bestseller of Romanian translations after 1989). M. Sebastian (1907 - 1944) has also been marginalized as a jew during the war but his literary work is now often reedited in Rumania and abroad.

\section{INDEX}

Thèmes : Histoire, Littérature roumaine, Histoire et littérature

Mots-clés : Banus Maria (1914-1999), Banus Maria (1914-1999), Transnistrie, juifs de Roumanie

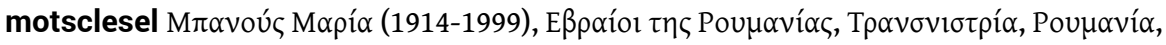

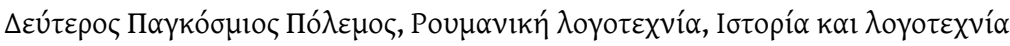
motsclestr Banuş Maria (1914-1999), Romanya'da Yahudiler, Transdinyester, Romanya, Ilk Dünya Savaşı, Romen edebiyatı, Tarih ve Edebiyat motsclesmk ЕВРЕИТЕ ВО РОМАНИЈА, ТРАНСНИСТРИЈА, РОМАНИЈА, ВТОРАТА СВЕТСКА ВОЈНА, РОМАНСКИ ЛИТЕРАТУРА, ИСТОРИЈА И ЛИТЕРАТУРА

Index géographique : Transnistrie, Roumanie

Keywords : Banus Maria (1914-1990), romanian Jews, Transnistria, Roumania, second World War, History, History and Literature

Index chronologique : guerre mondiale (1939-1945) 